\title{
Efficiency of Two Versions of SNOW against Guess \& Determine Attack
}

\author{
Muhammad Tahir ${ }^{1}$, Ghulam Mujtaba ${ }^{2}$, Saood Zia $^{1}$ and Tarranum $\mathrm{Naz}^{3}$ \\ ${ }^{1}$ Department of Computer Engineering, Sir Syed University. of Engineering \& \\ Technology Karachi, Pakistan \\ ${ }^{2}$ Department of Computer Science, Mohammad Ali Jinnah University, \\ Karachi, Pakistan \\ ${ }^{3}$ Department of Computer Science, Fatima Jinnah Women University Rawalpindi, \\ Pakistan \\ tahirfattani@gmail.com,gmujtabakorai@gmail.com,saood_zia@hotmail.com, \\ tarranum.rana@gmail.com
}

\begin{abstract}
This paper is about important field of cryptography which is stream ciphers. Stream ciphers are used to certify confidentiality on a communication channel. Many stream ciphers have been proposed during the history of cryptography. There are different types of stream ciphers e.g. RC4, Rabbit, Snow, in this paper the type of stream cipher will be discussed is 'SNOW'. There are three version of snow snow1.0, snow2.0 and modified version of snow, efficiency parameters of snow 2.0 and modified version of snow 2.0 will be discussed in this paper. In this paper efficiency of two version of SNOW have been analyzed against Guess and Determine Attack
\end{abstract}

Keywords: Guess and Determine Attack, snow1.0, snow2.0, Modified SNOW 2.0, Stream Cipher

\section{Introduction}

The more of our lives that we put online, the less privacy we have. And this is a problem that will just get worse over time. Computer communication needs to protect data during their transmission for secure transactions. The technique of traditional cryptography deals with simple algorithms and relied on very long keys. Stream ciphers are used for security [9] [14]. A cipher that makes use of an algorithmic procedure to produce an unending sequence of binary digits which is then combined either with plaintext to produce cipher text or with cipher text. The SNOW family belongs to word oriented stream cipher, which is based on Linear Feedback Shift register. SNOW 2.0 is an enhanced version of SNOW 1.0 claim to prevail over the weakness of SNOW 1.0. But later, some weaknesses found in SNOW 2.0 so another version which will called modified version of SNOW2.0 has been proposed which is claiming to overcome the weaknesses of SNOW2.0.

\subsection{Contributions}

Snow family belongs to word oriented stream ciphers and there are three versions of Snow, Snow1.0, Snow2.0 and modified version of Snow2.0. Three versions of SNOW are available which work efficiently against different attacks, so it is essential to check which one is more efficiently against guess and determine attack. The focus of the paper is to study efficiency parameters of SNOW2.0 \& modified version of SNOW2.0 against guess

Received (August 26, 2017), Review Result (December 15, 2017), Accepted (January 12, 2018) 
and determine attack. Guess and determine attack would applied on both versions of Snow 2.0 by using different number of keystreams i.e.32,128,256 and apply different time parameters on them and then,

- Find efficiency of both versions of SNOW against Guess and Determine Attack.

- Find that which version is more efficient against Guess and Determine attack.

\section{Preliminaries}

Different symbols are used in the working description which are define as below:

Addition by the symbol $\oplus$.

Addition modulo 232 by the symbol $\mathbf{⿴ 囗 十 .}$

Multiplication with $\alpha$ by $($.

A cyclic shift of 7 steps to the left by $\ll<$.

S-Box operation with s

\section{Description of Three Versions}

In this section brief description of three versions of snow is given.

\subsection{SNOW 1.0}

SNOW 1.0, originally simply SNOW, was submitted to the NESSIE project proposed by Patrik Ekdahl and Thomas Johansson [1]. The cipher works on 32-bit words and supports both 128- and 256-bit keys.

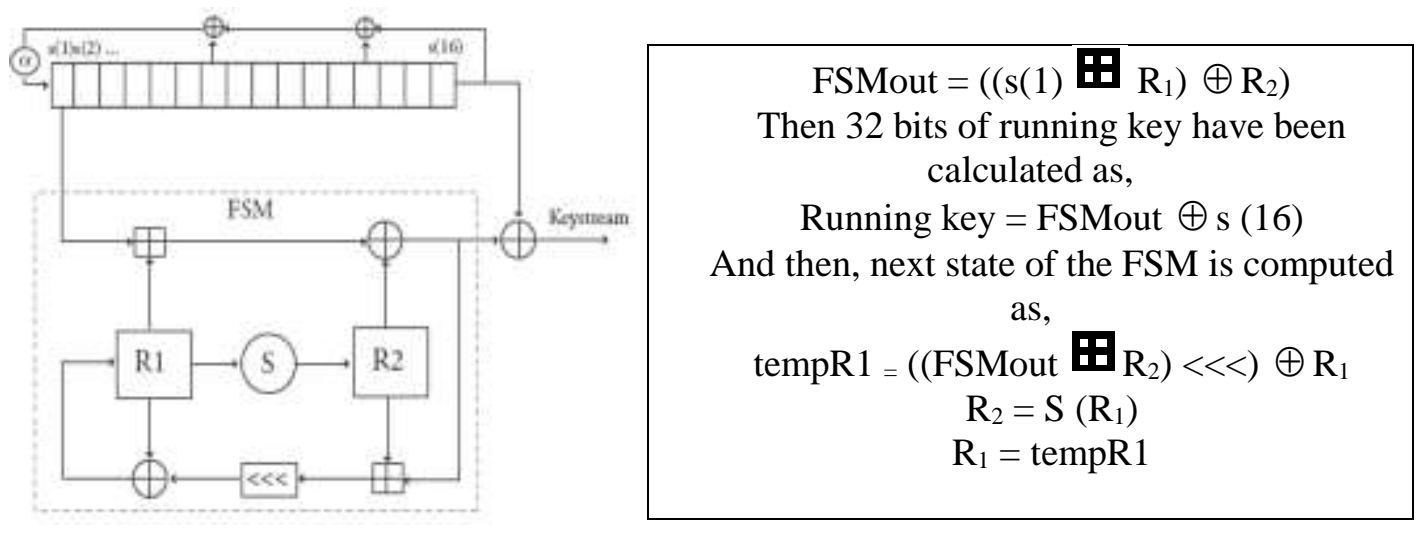

The LFSR has a primitive feedback polynomial,

$$
\mathrm{P}(\mathrm{x})=\mathrm{x}^{16} \oplus \mathrm{x}^{13} \oplus \mathrm{x}^{7} \oplus \alpha^{-1}
$$

The working of SNOW1.0 is explained below:

First of all Linear Feedback Shift Register and FSM registers $\left(\mathrm{R}_{1}\right.$ and $\left.\mathrm{R}_{2}\right)$ have been initialized. Then 32- bits (i.e. first register of LFSR) from LFSR is inserted into FSM as an input. Then the output of FSM is calculated as,

The S-Box, denoted by $\mathrm{S}(\mathrm{x})$, consists of four identical 8-to-8 bit S-Boxes and a permutation of the resulting bits [5]. 


\subsection{SNOW2.0 [3]}

SNOW2.0 is another version of Snow it uses word size of 32 bits.

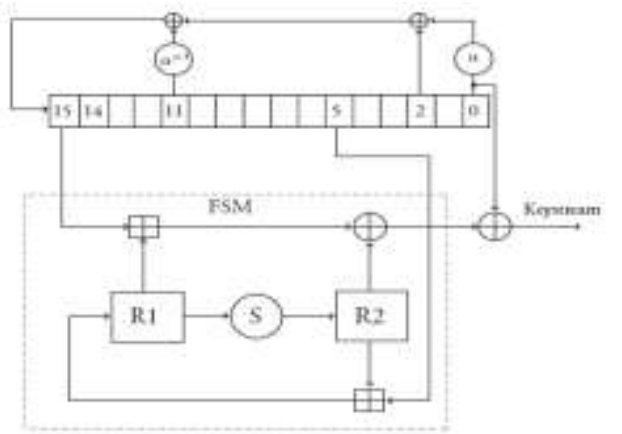

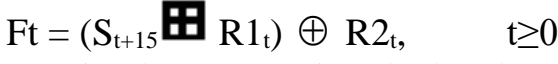

Then running key stream is calculated as,

$$
\mathrm{Zt}=\mathrm{f}_{\mathrm{t}} \oplus \mathrm{S}_{\mathrm{t}}, \quad \mathrm{t} \geq 1
$$

Then the cipher clocks and next state of the FSM is computed as,

$$
\begin{array}{ll}
\mathrm{R} 1_{\mathrm{t}+1}=\mathrm{S}_{\mathrm{t}+5} \mathbf{⿴ 囗 十}_{\mathrm{R} 2_{\mathrm{t}}} & \text { and } \\
\mathrm{R} 2_{\mathrm{t}+1}=\mathrm{S}\left(\mathrm{R} 1_{\mathrm{t}}\right) & \mathrm{t} \geq 0
\end{array}
$$

The LFSR has a primitive feedback polynomial,

$$
\mathrm{P}(\mathrm{x})=\alpha^{-1} x^{11} \oplus x^{2} \oplus \alpha x
$$

Working of SNOW2.0 is describe below:

First, Linear Feedback Shift Register (LFSR) is initialized, and registers of Finite State Machine (FSM) R1 and $\mathrm{R}_{2}$ are set to be zero.

The cipher clocks 32-times without producing any keystreams and incorporate output of FSM back into the LFSR. The main working of cipher has been started, and output of FSM has been computed as,

\subsection{Modified Version OF SNOW2.0 [4]}

The Modified Version of SNOW2.0 is improved version of Snow2.0 with minor changes, which are discuss below,

1. After XORing $\alpha$ (alpha) with $\mathrm{S}_{\mathrm{t}+2}$ take a circular left shift.

2. After XORing $\alpha^{-1}$ (alpha inverse) with $\mathrm{S}_{\mathrm{t}+11}$ take circular left shift again.

3. And take circular left shift of $\left(\mathrm{R} 1 \mathbf{⿴} \mathrm{S}_{\mathrm{t}+15}\right)$ once again before XORing with $\mathrm{R}_{2}$.

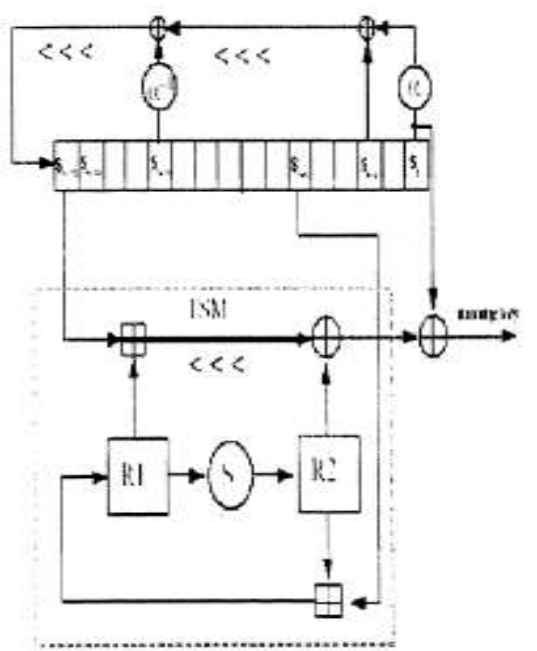

$$
\text { Tempft }=\mathrm{S}_{\mathrm{t}+15} \mathbf{H} \mathbf{R}_{\mathrm{t}}
$$

Take circular left shift of $\left(\mathrm{S}_{\mathrm{t}+15} \mathbf{H} \mathrm{R}_{\mathrm{t}}\right)$ before Xoring with $\mathrm{R}_{2}$

$$
\begin{aligned}
& \text { Tempft }=\left(\mathrm{S}_{\mathrm{t}+15} \mathbf{H}_{\left.\mathrm{R} 1_{\mathrm{t}}\right)} \ll<7\right) \\
& \mathrm{Ft}=\mathrm{tempf}_{\mathrm{t}} \oplus \mathrm{R} 2_{\mathrm{t}}, \quad \mathrm{t} \geq 0
\end{aligned}
$$

Then the running keystream is calculated as,

$$
\mathrm{Zt}=\mathrm{f}_{\mathrm{t}} \oplus \mathrm{S}_{\mathrm{t}}, \quad \mathrm{t} \geq 1
$$

The next state of FSM is computed as,

$$
\begin{array}{ll}
\mathrm{R} 1_{\mathrm{t}+1}=\mathrm{S}_{\mathrm{t}+5} \mathbf{⿴ 囗 十}_{\mathrm{R} 2_{\mathrm{t}}} & \text { and } \\
\mathrm{R} 2_{\mathrm{t}+1}=\mathrm{S}\left(\mathrm{R} 1_{\mathrm{t}}\right) & \mathrm{t} \geq 0
\end{array}
$$

And the next state of LFSR is calculated as, $S_{16}=\left(\left(\alpha^{-1} S_{\mathrm{t}+11}\right) \ll<, 7\right) \oplus S_{\mathrm{t}+2} \oplus\left(\left(\alpha S_{\mathrm{t}}\right) \ll<, 7\right)$

The LFSR has a primitive feedback polynomial,

$$
\mathrm{P}(\mathrm{x})=\alpha^{-1} x^{11} \oplus x^{2} \oplus \alpha x
$$


Working of Modified Version of SNOW 2.0 is described below.

- First, initialization is done in which Feedback Shift Register (LFSR) is initialized and two registers $\left(\mathrm{R}_{1}\right.$ and $\left.\mathrm{R}_{2}\right)$ of Finite State Machine (FSM) are set to be zero.

-Then clock operation is performed, the cipher will clock 32-times without producing any output. The output of FSM in incorporated back into the LFSR.

-After initialization and clocking, the main working of the cipher has been started, the input to the FSM ( $\mathrm{St}+5$ and $\mathrm{St}+15)$ are given and output of the FSM is calculated as,

\subsection{Research Methodology}

To achieve the final goal, the entire process is divided into following steps.

-Study, analysis and comparison of both versions of SNOW, and effect of guess and determine attack on both versions

-Apply Guess and Determine attack on both versions and analyze the time parameters.

- Compare the results of both versions, to illustrate conclusion that which model is more efficient against attack.

\section{Frame Work}

The main working of proposed system is to set time parameters in guess and determine attack's algorithm. The results of these parameters help in analyzing efficiency, these parameters are,

(I)User time (ii) Real time (iii) Start time (iv) End time (v) Elapsed time (vi) Clock tick per second.

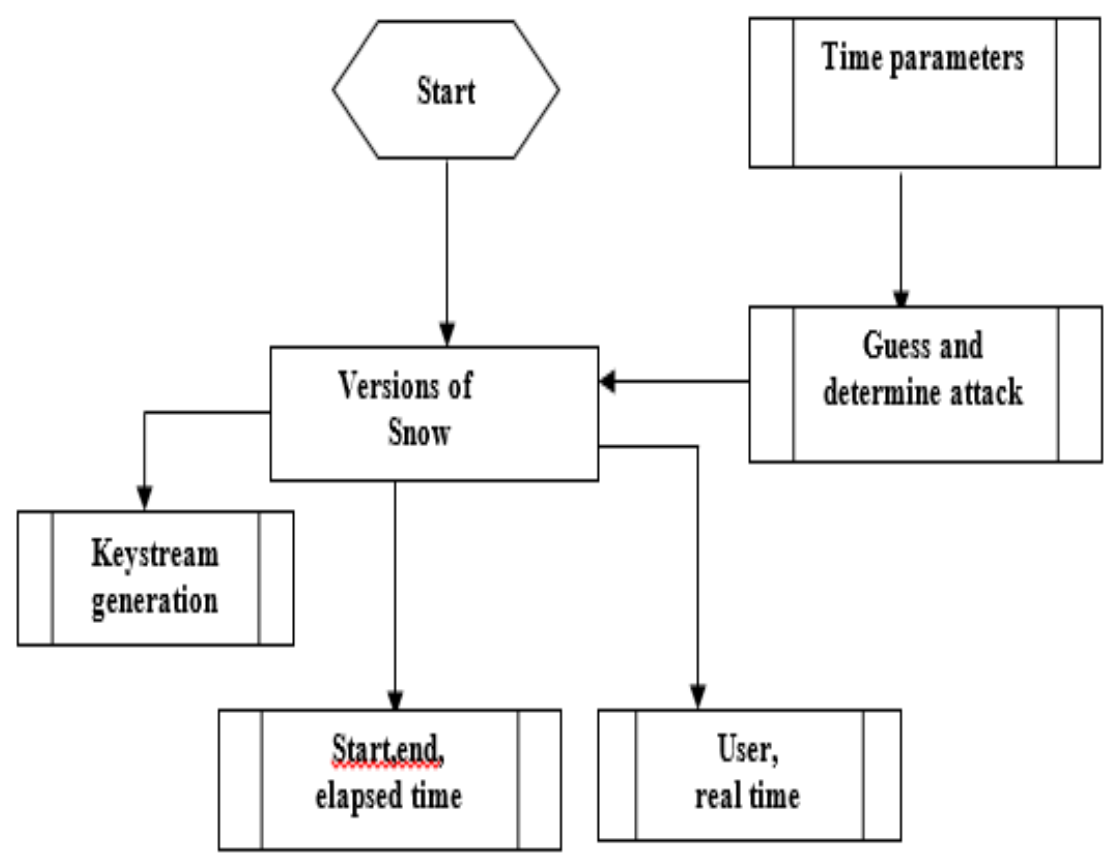

\section{Technique}

Following steps are used to obtain the results:

Step I: Apply Guess and determine attack on SNOW2.0 with 32, 128, 256 key streams. 
Step II: Note down the start time, end time, elapsed time, user time and real time of function (guess) with all these key streams.

Step III: Apply Guess and determine attack on modified version of SNOW2.0 with 32, 128, 256 key streams.

Step IV: Note down the start time, end time, elapsed time, user time and real time of function (guess) with all these key streams

Step V: Compare the user time and elapsed time of both versions with different key streams and analyzes which version has stable elapsed time.

\subsection{Comparison Table}

Comparison of timing parameters is shown in tables which will help to analyze the results

Table 5.1. Guess and Determine Attack on SNOW2.0 and Modified Version of SNOW 2.0 on 32-Bit Keystreams

\begin{tabular}{|c|c|c|c|c|}
\hline $\begin{array}{c}\text { No of } \\
\text { experiment }\end{array}$ & $\begin{array}{c}\text { User time } \\
\text { of } \\
\text { SNOW2.0 }\end{array}$ & $\begin{array}{c}\text { User time } \\
\text { of } \\
\text { Modified } \\
\text { version of } \\
\text { SNOW2.0 }\end{array}$ & $\begin{array}{c}\text { Elapsed time of } \\
\text { SNOW2 }\end{array}$ & $\begin{array}{c}\text { Elapsed time of } \\
\text { Modified version of } \\
\text { SNOW2.0 }\end{array}$ \\
\hline 1 & 0.4 & 0.3 & $3.8461538461538464 \mathrm{e}-01$ & $3.2967032967032966 \mathrm{e}-01$ \\
\hline 2 & 0.3 & 0.3 & $2.7472527472527475 \mathrm{e}-01$ & $3.2967032967032966 \mathrm{e}-01$ \\
\hline 3 & 0.3 & 0.8 & $3.2967032967032966 \mathrm{e}-01$ & $8.7912087912087911 \mathrm{e}-01$ \\
\hline 4 & 0.3 & 0.3 & $2.7472527472527475 \mathrm{e}-01$ & $2.7472527472527475 \mathrm{e}-01$ \\
\hline 5 & 0.4 & 0.3 & $3.8461538461538464 \mathrm{e}-01$ & $3.2967032967032966 \mathrm{e}-01$ \\
\hline 6 & 0.2 & 0.3 & $2.1978021978021978 \mathrm{e}-01$ & $2.7472527472527475 \mathrm{e}-01$ \\
\hline 7 & 0.3 & 0.3 & $3.2967032967032966 \mathrm{e}-01$ & $2.7472527472527475 \mathrm{e}-01$ \\
\hline 8 & 0.3 & 0.3 & $2.7472527472527475 \mathrm{e}-01$ & $2.7472527472527475 \mathrm{e}-01$ \\
\hline 9 & 0.3 & 0.3 & $3.2967032967032966 \mathrm{e}-01$ & $2.7472527472527475 \mathrm{e}-01$ \\
\hline 10 & 0.3 & 0.3 & $2.7472527472527475 \mathrm{e}-01$ & $2.7472527472527475 \mathrm{e}-01$ \\
\hline
\end{tabular}

Table 5.2. Guess and Determine Attack on SNOW 2.0 and Modified Version of SNOW2.0 on 128-Bit Keystreams

\begin{tabular}{|c|c|c|c|c|}
\hline $\begin{array}{c}\text { No of } \\
\text { experiment }\end{array}$ & $\begin{array}{c}\text { User time } \\
\text { of } \\
\text { SNOW2.0 }\end{array}$ & $\begin{array}{c}\text { User time of } \\
\text { Modified } \\
\text { version of } \\
\text { SNOW2.0 }\end{array}$ & $\begin{array}{c}\text { Elapsed time of } \\
\text { SNOW2.0 }\end{array}$ & $\begin{array}{c}\text { Elapsed time of } \\
\text { Modified version of } \\
\text { SNOW2.0 }\end{array}$ \\
\hline 1 & 0.4 & 0.1 & $3.8461538461538464 \mathrm{e}-01$ & $1.0989010989010989 \mathrm{e}-01$ \\
\hline 2 & 0.5 & 0.4 & $5.4945054945054950 \mathrm{e}-01$ & $4.3950643956043955 \mathrm{e}-01$ \\
\hline 3 & 0.3 & 0.3 & $2.7472527472527475 \mathrm{e}-01$ & $2.7472527472527475 \mathrm{e}-01$ \\
\hline 4 & 0.4 & 0.4 & $3.8461538461538464 \mathrm{e}-01$ & $3.8484615384615384 \mathrm{e}-01$ \\
\hline 5 & 0.3 & 0.3 & $2.7472527472527475 \mathrm{e}-01$ & $2.7472527472527475 \mathrm{e}-01$ \\
\hline 6 & 0.3 & 0.3 & $2.7472527472527475 \mathrm{e}-01$ & $3.2967032967032966 \mathrm{e}-01$ \\
\hline 7 & 0.2 & 0.3 & $2.1978021978021978 \mathrm{e}-01$ & $3.2967032967032966 \mathrm{e}-01$ \\
\hline 8 & 0.2 & 0.3 & $1.6483516483516483 \mathrm{e}-01$ & $3.2967032967032966 \mathrm{e}-01$ \\
\hline 9 & 0.4 & 0.3 & $3.8461538461538464 \mathrm{e}-01$ & $3.2967032967032966 \mathrm{e}-01$ \\
\hline 10 & 0.3 & 0.3 & $2.7472527472527475 \mathrm{e}-01$ & $3.2967032967032966 \mathrm{e}-01$ \\
\hline
\end{tabular}




\section{Table 5.3. Guess and Determine Attack on SNOW2.0 and Modified Version} of SNOW2.0 on 256-Bit Keystreams

\begin{tabular}{|c|c|c|c|c|}
\hline $\begin{array}{c}\text { No of } \\
\text { experiment }\end{array}$ & $\begin{array}{c}\text { User time } \\
\text { of } \\
\text { SNOW2.0 }\end{array}$ & $\begin{array}{c}\text { User time of } \\
\text { Mod } \\
\text { version of } \\
\text { SNOW2.0 }\end{array}$ & $\begin{array}{c}\text { Elapsed time of } \\
\text { SNOW2 }\end{array}$ & $\begin{array}{c}\text { Elapsed time of } \\
\text { Modified version of } \\
\text { SNOW2.0 }\end{array}$ \\
\hline 1 & 0.3 & 0.4 & $2.7472527472527475 \mathrm{e}-01$ & $3.8461538461538464 \mathrm{e}-01$ \\
\hline 2 & 0.3 & 0.3 & $3.2967032967032966 \mathrm{e}-01$ & $3.2967032967032966 \mathrm{e}-01$ \\
\hline 3 & 0.4 & 0.3 & $3.8484615384615384 \mathrm{e}-01$ & $3.2967032967032966 \mathrm{e}-01$ \\
\hline 4 & 0.4 & 0.3 & $3.8484615384615384 \mathrm{e}-01$ & $3.2967032967032966 \mathrm{e}-01$ \\
\hline 5 & 0.2 & 0.3 & $2.1978021978021978 \mathrm{e}-01$ & $3.2967032967032966 \mathrm{e}-01$ \\
\hline 6 & 0.3 & 0.5 & $2.7472527472527475 \mathrm{e}-01$ & $1.6483516483516483 \mathrm{e}-01$ \\
\hline 7 & 0.3 & 0.3 & $2.7472527472527475 \mathrm{e}-01$ & $3.8461538461538464 \mathrm{e}-01$ \\
\hline 8 & 0.3 & 0.3 & $2.7472527472527475 \mathrm{e}-01$ & $4.9450549450549452 \mathrm{e}-01$ \\
\hline 9 & 0.3 & 0.3 & $2.7472527472527475 \mathrm{e}-01$ & $3.2967032967032966 \mathrm{e}-01$ \\
\hline 10 & 0.3 & 0.4 & $2.7472527472527475 \mathrm{e}-01$ & $3.8461538461538464 \mathrm{e}-01$ \\
\hline
\end{tabular}

\section{Experimental Results}

Guess and determine attack is applied on diverse number of key streams i.e. 32,128 and 256, the efficiency of both versions against GD attack is measured by determining different time parameters of algorithm i.e. user time, real time, start time, end time and elapsed time, the version which show consistent or stable user time and elapsed time, that version will be more efficient against Guess and determine attack. The experimental phase includes three sub phases.

Phase I: In phase I Guess and Determine attack will applied on both versions with 32bit keystreams, this experiment is applied 10 times, in each experiment, clock tick per second, start time, end time, elapsed time, user time, and real time has been noted.

Phase II: In phase II Guess and Determine attack will applied on both versions with 128-bit keystreams, this experiment is applied 10 times, in each experiment, clock tick per second, start time, end time, elapsed time, user time, real time has been noted.

Phase III: In phase III Guess and Determine attack will applied on both versions with 256-bit keystreams, this experiment is applied 10 times, in each experiment, clock tick per second, start time, end time, elapsed time, user time, real time has been noted.

Before analyzing all three phases of both versions it is compulsory to give the results of experiment.

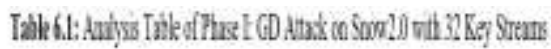

\begin{tabular}{|c|c|c|c|c|c|}
\hline $\begin{array}{l}\text { tin } \\
\text { int }\end{array}$ & $\begin{array}{l}\text { bad } \\
\text { bx }\end{array}$ & sentwe & Intix & Elypdtar & $\begin{array}{l}\text { Cowis } \\
\text { par ind }\end{array}$ \\
\hline Qt. & 10 & 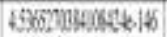 & $4560 \times 454046$ & 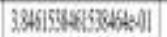 & 13107 \\
\hline 01 & 40 & 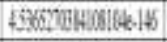 & 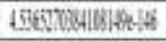 & 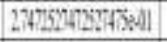 & $1310 \%$ \\
\hline 03 & 40 & 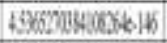 & 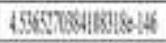 & 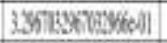 & 1300 \\
\hline 03 & (10) & 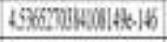 & 4565) & 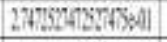 & 190 \\
\hline Qt & 40 & 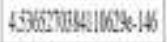 & 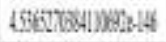 & 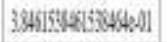 & 13160 \\
\hline Q2 & II) & 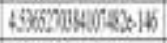 & 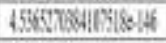 & 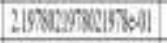 & 130 \\
\hline 03 & 10 & 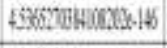 & 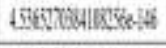 & 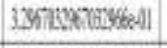 & 1310 \\
\hline 03 & 10 & 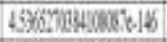 & 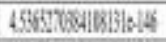 & $24+2245 \times 4$ 에 & 1306 \\
\hline 03 & 1i) & 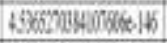 & 456sosulnowello & 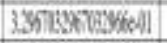 & 1910 \\
\hline 01 & (10) & 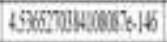 & 65E. & 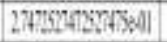 & 1310 \\
\hline
\end{tabular}

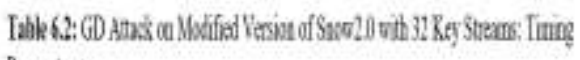
Praniters

\begin{tabular}{|c|c|c|c|c|c|}
\hline $\begin{array}{l}\text { Ist } \\
\text { tar }\end{array}$ & $\begin{array}{l}\text { Dal } \\
\text { tat }\end{array}$ & Surt tax & Evity & Elapetibe & $\begin{array}{l}\text { Coutin } \\
\text { perwol }\end{array}$ \\
\hline [1] & 11 & 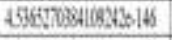 & 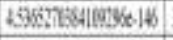 & 3XG) & $53 \pi$ \\
\hline [3] & II & 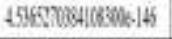 & 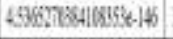 & 3.6n & $31 n$ \\
\hline II & 01 & 465:Y0estossis-145 & 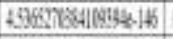 & 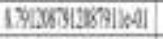 & Bin \\
\hline H & $\Delta$ & 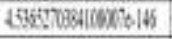 & 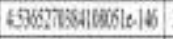 & $2 x+25075245 \times 1$ & 1397 \\
\hline W & Bb & 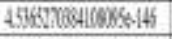 & 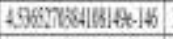 & 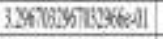 & $\mathrm{B} / \mathrm{N}$ \\
\hline 4 & 61 & 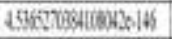 & 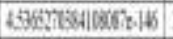 & 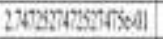 & $13 \pi$ \\
\hline Q & QS & 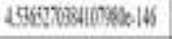 & 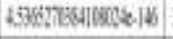 & 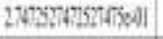 & 1395 \\
\hline W & $\Delta$ & 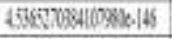 & 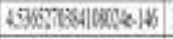 & 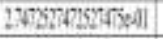 & BIT: \\
\hline [1] & 4 & 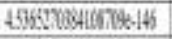 & 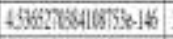 & 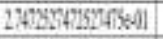 & 3 III \\
\hline b] & bs & 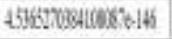 & 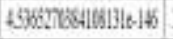 & 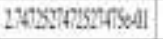 & $B \mathrm{II}^{\circ}$ \\
\hline
\end{tabular}




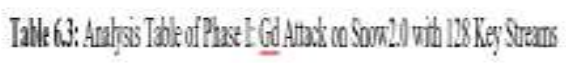

\begin{tabular}{|c|c|c|c|c|c|}
\hline $\begin{array}{l}\text { Ist } \\
\text { tut }\end{array}$ & $\begin{array}{l}\text { Bal } \\
\text { int }\end{array}$ & Surtici & batits: & Elyod fur & $\begin{array}{l}\text { Cotifit } \\
\text { perend }\end{array}$ \\
\hline If & 00 & 4567) & 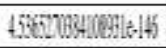 & 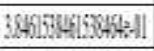 & 1317 \\
\hline 15 & 00 & 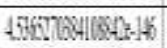 & 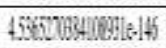 & 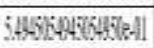 & B1II \\
\hline 13 & 00 & 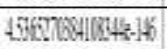 & 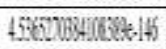 & 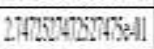 & B1] \\
\hline IS & 00 & 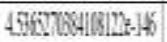 & 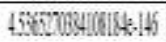 & 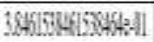 & Bin \\
\hline 13 & 00 & 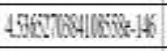 & 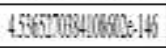 & 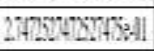 & B17 \\
\hline 43 & 60 & 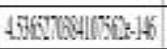 & 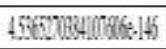 & 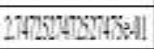 & Bin \\
\hline 12 & 00 & 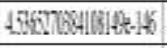 & 4566062-6614t:15 & 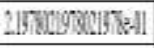 & $13 \pi$ \\
\hline 12 & 00 & 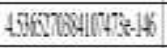 & 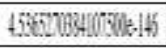 & 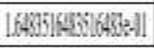 & 5137 \\
\hline It & 000 & 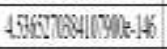 & 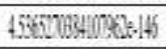 & 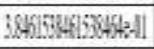 & 312 \\
\hline 13 & 10 & $45670 \times 41(332-14)$ & 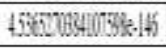 & 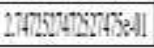 & 1312 \\
\hline
\end{tabular}

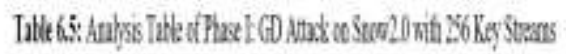

\begin{tabular}{|c|c|c|c|c|c|}
\hline $\begin{array}{l}\text { tie } \\
\text { te }\end{array}$ & $\begin{array}{l}\mathrm{Bd} \\
\mathrm{int}\end{array}$ & sartat & Evithe & Aqudter & $\begin{array}{l}\text { Odist } \\
\text { permod }\end{array}$ \\
\hline 05 & II & 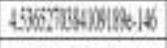 & 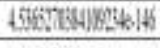 & 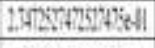 & $131 \pi$ \\
\hline 03 & it & 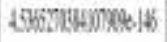 & 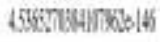 & 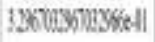 & 13:5 \\
\hline 04 & of & 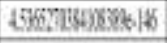 & 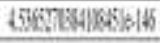 & 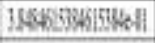 & $13 \pi$ \\
\hline Q4 & ll & 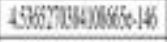 & 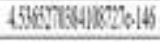 & 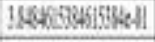 & [3] \\
\hline Q2. & II & 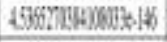 & 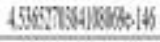 & 2monsmused & $193 \pi$ \\
\hline 05 & (1) & 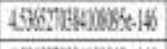 & 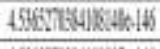 & 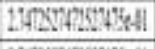 & $13: 7$ \\
\hline Q3 & QI & 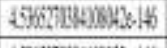 & 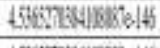 & 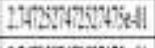 & $19 \pi$ \\
\hline 13 & a & 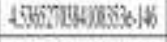 & 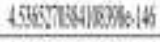 & 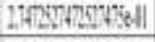 & $19 M$ \\
\hline D3 & II & 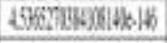 & 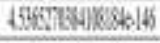 & 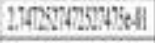 & 1397 \\
\hline 25 & if & 4502 H. & 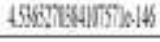 & 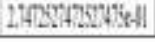 & 13137 \\
\hline
\end{tabular}

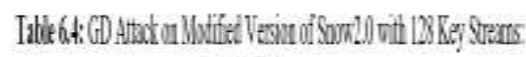
Inimigraratos

\section{Evaluation of Results}

After the performing different experiments, the next step is evaluation of results which had been obtained from experiments this is the most important and major step in testing of the proposed system. The whole experimental work comprises on three phases phase I, phase II and phase III, their results will be shown above; now the results of all three phases will be evaluated. The following subsection will contain the evaluation of results on basis of these experiments.

\subsection{Evaluation of Phase I and Graphical Representation}

In Phase I, 10 experiments are performed on both versions. Each experiment is applied 10 times each time the algorithm will give different guess values and keystreams and different values of timing parameters. The experimental results on SNOW2.0 with 32-bit keystreams shows the values of timing parameters which are not stable there is variation in its user time, and elapsed time their results are not consistent, on the other hand same experiment is applied on modified version of SNOW2.0 with 32-bit key streams, its results shows consistency in its timing parameters which means that user time and elapsed time becomes stable after some time. This concludes that modified version of SNOW2.0 will be more efficient than original SNOW 2.0 against guess and Determine Attack Comparison table of user time and elapsed time of both versions is shown below to understand the conclusion more clearly. 
Table 7.1: Analysis results of both versions

\begin{tabular}{|c|c|c|c|c|}
\hline $\begin{array}{l}\text { No of } \\
\text { erperiment }\end{array}$ & $\begin{array}{l}\text { User time } \\
\text { of } \\
\text { siow2. }\end{array}$ & $\begin{array}{l}\text { User time of } \\
\text { Yodified } \\
\text { rersion of } \\
\text { SNOW2:0 }\end{array}$ & $\begin{array}{l}\text { Elyysed time of } \\
\text { SNOW: }\end{array}$ & $\begin{array}{l}\text { Elaped time of } \\
\text { Modified rersion of } \\
\text { SxOT20 }\end{array}$ \\
\hline 1 & 0.4 & 03 & $3.8461539461538464 \mathrm{e}-01$ & $3.2967032967032966 \mathrm{e}-01$ \\
\hline 2 & 0.3 & 0.3 & $2.7472527472527475 \mathrm{e}-01$ & $3.2967032967032966 \mathrm{e}-01$ \\
\hline 3 & 0.3 & 0.8 & $3.2967032967032966 \mathrm{e}-01$ & 8.7912087912087911e-01 \\
\hline 4 & 0.3 & 0.3 & $2.7472527472527475 \mathrm{e}-01$ & $2.7472527472527475 \mathrm{e}-01$ \\
\hline 5 & 0.4 & 0.3 & $3.8461538461538464 \mathrm{e}-01$ & $3.29670329670329666-01$ \\
\hline 6 & 0.2 & 03 & $2.1978021978021978 \mathrm{e}-01$ & $2.7472527472527475 \mathrm{e}-01$ \\
\hline 7 & 0.3 & 03 & $32967032967032966 \mathrm{e}-01$ & $2.742527472527475 \mathrm{e}-01$ \\
\hline 8 & 0.3 & 03 & $2.7472527472527475 \mathrm{e}-01$ & $2.7472527472527475 \mathrm{e}-01$ \\
\hline 9 & 0.3 & 03 & $32967032967032966 \mathrm{e}-01$ & $2.7472527472527475 \mathrm{e}-01$ \\
\hline 10 & 0.3 & 03 & $2.7472527472527475 \mathrm{e}-01$ & $2.7472527472527475 \mathrm{e}-01$ \\
\hline
\end{tabular}

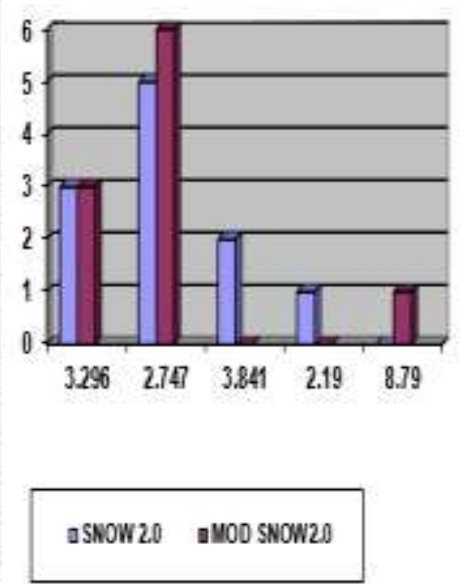

Both table and graphical representation shows that elapsed time and user time of SNOW2.0 is not stable, it has been changed after every iteration, but SNOW2.0 shows stability for elapsed time results after fifth iteration and user time remains stable during all experiments, which means it is more efficient than SNOW 2.0

\subsection{Evaluation of Phase II and Graphical Representation:}

In Phase II, 10 experiments are performed on both versions. Each experiment is applied 10 times each time the algorithm will give different guess values and keystreams and different values of timing parameters. The experimental results on SNOW2.0 with 128-bit keystreams shows the values of timing parameters, which are not stable there is variation in its user time and elapsed time, their results are not consistent, on the other hand same experiment is applied on modified version of SNOW2.0 with 128 bit key streams, its results show consistency in its timing parameters which means that user time and elapsed time becomes stable after some experiments. This concludes that modified version of SNOW2.0 will be more efficient than original SNOW 2.0 against guess and Determine attack.

Comparison table of user time and elapsed time of both versions is shown below to understand the conclusion more clearly.

Table 72: Aarysis results of both nersicos

\begin{tabular}{|c|c|c|c|c|}
\hline $\begin{array}{l}\text { No of } \\
\text { eqperimeat }\end{array}$ & $\begin{array}{l}\text { Ther time } \\
\text { of } \\
\text { sxow2 }\end{array}$ & 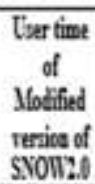 & $\begin{array}{l}\text { Elapeed time of } \\
\text { sNow: }\end{array}$ & $\begin{array}{l}\text { Elopsed time of } \\
\text { Modified renios of } \\
\text { SToTI20 }\end{array}$ \\
\hline 1 & 0.4 & 0.1 & $3.8461539461539464=01$ & $109990109990109996-1]$ \\
\hline$?$ & 0.5 & 04 & 5.4943034945036950401 & 4397664356045355601 \\
\hline 3 & 0.3 & 03 & $27+2521472524778601$ & $2747252472527475 \mathrm{a}-1$ \\
\hline 4 & 0.4 & 04 & $3.4615346153444=01$ & $3844615394615344+01$ \\
\hline 5 & 0.3 & 0.3 & $27472527+72527475601$ & $27472527472527456-11$ \\
\hline 6 & 0.3 & 0.3 & $2747522+72522473=01$ & 32867032967033666001 \\
\hline 7 & 0.2 & 03 & $21973019 \times 202197 \mathrm{~K}_{\mathrm{E}} 01$ & $389603286703866 \mathrm{e} 01$ \\
\hline 8 & 0.2 & 0.3 & $1.6485516485531 .4835-01$ & $3.9673286003866 \mathrm{a}$ \\
\hline 9 & 0.4 & 0.3 & $3.46158446152444+01$ & $328003286033866 \mathrm{a} 01$ \\
\hline 10 & 03 & 03 & $274252742527473 \mathrm{k}=1$ & $32967032560032966 \mathrm{e} 01$ \\
\hline
\end{tabular}

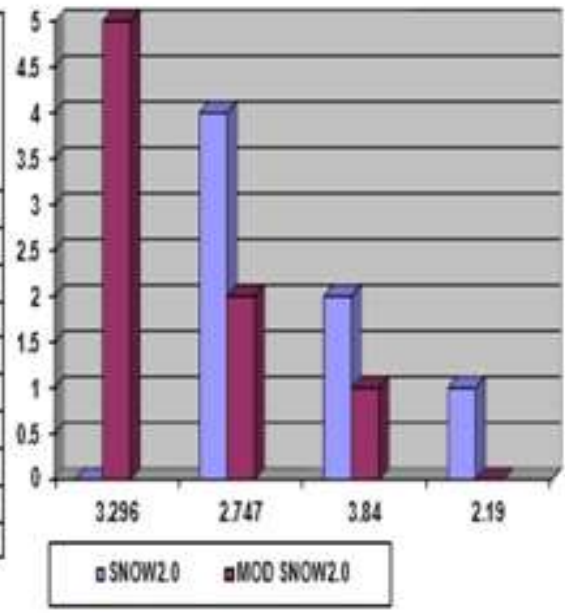


Above table and graphical representation shows that elapsed time and user time of SNOW2.0 will not stable it has been changed after every iteration, but user time and elapsed time of modified version of SNOW2.0 shows stability in elapsed time results after fifth iteration and user time will become stable after fourth experiments, which means that modified version of SNOW2.0 is more efficient than SNOW 2.0

\subsection{Evaluation of Phase III and Graphical Representation}

In Phase III, 10 experiments are performed on both versions. Each experiment is applied 10 times each time the algorithm will give different guess values and keystreams and different values of timing parameters. The experimental results on modified SNOW2.0 with 256-bit key streams shows the values of timing parameters, which are not stable, there is variation in its user time and elapsed time their results are not consistent, on the other hand same experiment is applied on SNOW2.0 with 256-bit key streams, its results show consistency in its timing parameters which means that user time and elapsed time becomes stable after some experiments. This concludes that if no of keystreams is large e.g. 256 than SNOW2.0 will be more efficient than modified version of SNOW 2.0 against guess and Determine attack. Comparison table of user time and elapsed time of both versions is shown below to understand the conclusion more clearly.

Table 7.3: Analygis results of both versions

\begin{tabular}{|c|c|c|c|c|}
\hline $\begin{array}{c}\text { No of } \\
\text { experimen } \\
t\end{array}$ & $\begin{array}{c}\text { User time } \\
\text { of } \\
\text { SNOW2.0 }\end{array}$ & $\begin{array}{c}\text { User time of } \\
\text { Modified version } \\
\text { of SNOW2.0 }\end{array}$ & $\begin{array}{c}\text { Elapsed time of } \\
\text { SNOW2 }\end{array}$ & $\begin{array}{c}\text { Elapsed time of } \\
\text { Modified rersion of SNOW2.0 }\end{array}$ \\
\hline 1 & 0.3 & 0.4 & $2.7472527472527475 \mathrm{e}-0 !$ & $3.8461538461533464 \mathrm{e}-01$ \\
\hline 2 & 0.3 & 0.3 & $3.2967032967032966 \mathrm{e}-0 !$ & $3.2967032967032966 \mathrm{e}-01$ \\
\hline 3 & 0.4 & 0.3 & $3.8484615384615334 \mathrm{e}-0 !$ & $3.2967032967032966 \mathrm{e}-01$ \\
\hline 4 & 0.4 & 0.3 & $3.8484515384615384 \mathrm{e}-0 !$ & $3.2967032967032966 \mathrm{e}-01$ \\
\hline 5 & 0.2 & 0.3 & $2.1978021978021978 \mathrm{e}-0 !$ & $3.2967032967032966 \mathrm{e}-01$ \\
\hline 6 & 0.3 & 0.5 & $2.7472527472527475 \mathrm{e}-01$ & $1.6483516483516483 \mathrm{e}-01$ \\
\hline 7 & 0.3 & 0.3 & $2.7472527472527475 \mathrm{e}-0 !$ & $3.8461538461538464 \mathrm{e}-01$ \\
\hline 8 & 0.3 & 0.3 & $2.7472527472527475 \mathrm{e}-0 !$ & $4.9450549450549452 \mathrm{e}-01$ \\
\hline 9 & 0.3 & 0.3 & $2.7472527472527475 \mathrm{e}-0 !$ & $3.2967032967032966 \mathrm{e}-01$ \\
\hline 10 & 0.3 & 0.4 & $2.7472527472527475 \mathrm{e}-0 !$ & $3.8461538461538464 \mathrm{e}-01$ \\
\hline
\end{tabular}

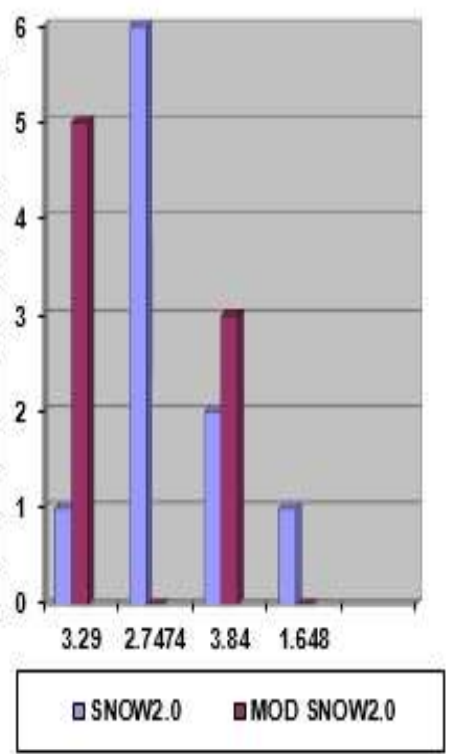

Above table and graphical representation shows that elapsed time and user time of SNOW2.0 will not stable it has been changed after every iteration, but user time and elapsed time of modified version of SNOW2.0 shows stability for elapsed time results after fifth iteration and user time will become stable after fourth experiments, which means that modified version of SNOW2.0more efficient than SNOW2.0.

\section{Conclusion and Future Work}

\subsection{Conclusion}

Guess and determine attack has been applied on two versions of SNOW (i.e. Original SNOW 2.0 and Modified Version of SNOW 2.0). The attack consists of three phases. Phase I and Phase II conclude that, for small number of keystreams modified version of SNOW 2.0 is more efficient against Guess and Determine attack, whereas Phase III concluded that, for large number of keystreams, SNOW 2.0 is more efficient. From 
analysis and results it is concluded that if the plaintext has converted into cipher text is in small amount then Modified Version of SNOW 2.0 should be used, on the other hand if large amount of plain text has to encrypt the SNOW2.0 should be used.

\subsection{Future Work}

All the experimentation will be done by using static Linear Feed Back Shift register; the use of static values may not give desired results, so the use of dynamic values may fulfill the requirements of cryptanalysis.

\section{References}

[1] Patrik Ekdahl,Thomas Johnsson "A new version of stream cipher SNOW" dept of information technology Lund University,Sweden.

[2] Philip Hawkes and Gregory G.rose "Guess-and-Determine Attack on SNOW".

[3] Dai watanabe,Alex Biryukuv "A Distinguishing attack of SNOW 2.0"

[4] Hadi Ahmadi 1, Taraneh Eghlidos 2, Shahram Khazaei 3 "Improved Guess and Determine Attack SOSEMANUK” School of Electrical Engineering, Sharif University of Technology, Tehran, Iran

[5] http://en.wikipedia.org/wiki/LFSR

[6] U.S National Institute of standards and technology. 12 may 2008.Available from: http://www.nist.gov/dads/HTML/finite state machine.html

[7] http://en.wikipedia.org/wiki/cryptography

[8] http://www.tech-faq.com/block-stream- ciphers.html

[9] Stream cipher for message confidentiality M.Ismail Jabiullah and M Lutfar Rahman,Depratment of computer science and engineering university of Dhakha.

[10] Applied stream cipher in mobile communications B.Eng. in Computer Science \& Technology, Beijing Polytechnic University Chine, et de nationalit_e chinoise

[11] http://homepage.mac.com/afj/lfsr.html

[12] Alexander Maximov, "Some Words on Cryptanalysis of Stream Ciphers", Ph.D. Thesis, Lund University, 2006.

[13] http://www2.cs.uidaho.edu/ rinker/cs113/BigO.pdf

[14] "Analysis of LFSR Based Snow Family against Guess and Determine Attack" Authors: Aihab Khan Tarranum Naz, Muhammad Tahir Journal of Basic and Applied Scientific Research, 2013

\section{Authors}

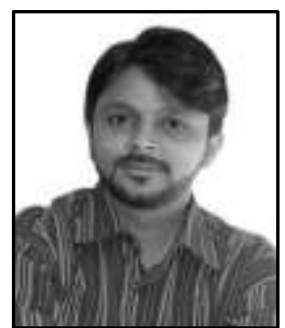

Muhammad Tahir, he received the BS degree in computer engineering from Sir Syed University, M.E. degree in Computer System from NED University and PhD in Information Science from University of Roma Tor Vergata. He is currently Associate Professor in Sir Syed University of Engineering and Technology, Karachi. His research interests include IP Switches/Routing, IPv4 Protocol, Firewall, IoT, Security Cryptography and Wireless Networks.

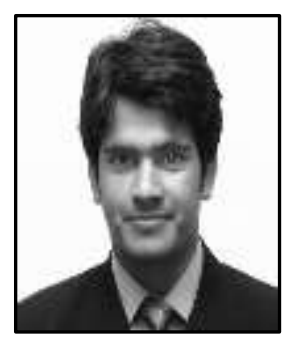

Ghulam Mujtaba, he received in BS Computer Sciences (20092013) from COMSATS Institute of Information Technology (CIIT), Lahore, Pakistan's top most ranked university in Computer Science and 401-500 in QS World University Rankings in Computer Science and IT on ICT R\&D Fully funded scholarship by ministry of IT, Pakistan and MS Computer Science (2014 - 2016) from Indus University, Karachi, Pakistan. His research interests include mobile computing, Human Computer Interaction, game theory and Security and Cryptography. 


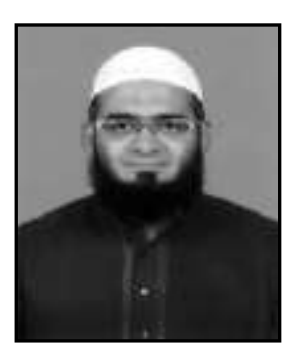

Syed Saood Zia, he obtained his BSc (Hons) in Computing \& Information Systems from London Metropolitan University in the year of 1999. He has completed his Master of Computer Science from KASBIT in 2004 and MS in Mobile Computing \& Information Systems from Hamdard University in 2006. He has recently completed his PhD in the field of Information Technology in 2017. $\mathrm{He}$ has more than seventeen years of teaching experience in different academic institutions. He is working as Assistant Professor in Computer Engineering Department at SSUET. He is a member of British computer society. His research area is knowledge based systems, Information retrieval, Big Data Analytics, Sentiment Analysis, Opinion Mining and Data Mining.

Tarranum Naz, she is a graduate from Dept. Computer Science, Fatima Jinnah Women University Pakistan. She is an active researcher in the field of information security and cryptography in Computer Sciences Dept. Fatima Jinnah Women University, Pakistan. She can be contacted at tarranumpk@yahoo.com, Fatima Jinnah Women University, Rawalpindi Pakistan. 
International Journal of Security and Its Applications

Vol. 12, No. 1 (2018) 